\title{
Larks, owls, swifts and woodcocks among the fruit flies: heritable individual differences in the sleep-wake pattern induced by long, and sometimes, hot Siberian summer days
}

\author{
L.P. Zakharenko ${ }^{1 *}$, D.V. Petrovsky ${ }^{1}$, A.A. Putilov ${ }^{2}$ \\ ${ }^{1}$ Institute of Cytology and Genetics SB RAS, Novosibirsk, Russia \\ ${ }^{2}$ Institute of Molecular Biology and Biophysics, Novosibirsk, Russia \\ *e-mail: zakharlp@bionet.nsc.ru
}

Key words: morning-evening preference; chronotype; circadian rhythm; photoperiod; temperature; locomotor activity; morning and evening oscillators; migration out of Africa

Motivation and Aim: Drosophila melanogaster and our own species have many things in common including history of rapid out-of-Africa dispersal. In Eurasia, we both faced the problem of adjustment of our circadian rhythms to seasonal variation in day length, and each of us usually responds to night sleep disturbances by prolongation of siesta. To further explore similarity between two species, a division of flies into chronotypes was examined.

Methods: By testing the circadian rhythms of locomotor activity in constant darkness, 4 strains originating from three wild populations of Africa, Europe and the USA were selected as the representatives of 4 distinct chronotypes [1]: "larks" (early morning and evening peaks), "owls" (late morning and evening peaks), "swifts" (early morning and late evening peaks) and "woodcocks" (late morning and early evening peaks). The locomotor rhythms and sleep-wake pattern of selected chronotypes were tested under either long day condition (L:D 20:4) at $20^{\circ} \mathrm{C}$ or combination of high temperature $\left(29^{\circ} \mathrm{C}\right)$ with L:D 20:4.

Results: Despite identity of such an experimental condition for 4 chronotypes, their circadian rhythms showed 4 distinct patterns of maladaptive response to it. For instance, as we expected for L:D 20:4, the flies of any chronotype failed to increase the phase angle between morning and evening peaks of activity beyond 16 hours (i.e., to remain in synch with transitions from darkness to light and back from light to darkness that were divided by 20 -h interval). Instead, "woodcocks" became fully arrhythmic. "Owls" exhibited as many as three prominent peaks of activity. In "larks" the reaction to the light off was blunted but their response to the light switch was very strong, while the opposite was true for "swifts".

Conclusion: An experimental study of heritable chronotypes in the fruit fly can deepen our understanding the genetic underpinnings of individual variation in vulnerability to maladaptive sleep-wake behavior, circadian misalignment and sleep disorders.

Acknowledgements: LPZ and DVP were partially funded by the Federal Research Program (the project number 0324-2018-0016).

\section{Reference}

1. Putilov A.A. (2017) Owls, larks, swifts, woodcocks and they are not alone: A historical review of methodology for multidimensional self-assessment of individual differences in sleep-wake pattern. Chronobiol Int. 34:426-437. 